\title{
Spermidine/spermine- $N^{1}$-acetyltransferase-2 (SSAT2) acetylates thialysine and is not involved in polyamine metabolism
}

\author{
Catherine S. COLEMAN*1 Bruce A. STANLEY $\uparrow$, A. Daniel JONES $\ddagger$ and Anthony E. PEGG* \\ *Department of Cellular and Molecular Physiology, The Milton S. Hershey Medical Center, Pennsylvania State University College of Medicine, P.0. Box 850, Hershey, PA 17033, U.S.A., \\ $\uparrow$ Section of Research Resources, The Milton S. Hershey Medical Center, Pennsylvania State University College of Medicine, P.0. Box 850, Hershey, PA 17033, U.S.A., and \\ †Department of Chemistry, Pennsylvania State University, University Park, PA 16082, U.S.A.
}

\begin{abstract}
Spermidine/spermine- $N^{1}$-acetyltransferase (SSAT1) is a shortlived polyamine catabolic enzyme inducible by polyamines and polyamine analogues. Induction of SSAT1 plays an important role in polyamine homoeostasis, since the $N^{1}$-acetylated polyamines can be excreted or oxidized by acetylpolyamine oxidase. We have purified a recombinant human acetyltransferase (SSAT2) that shares $45 \%$ identity and $61 \%$ homology with human SSAT1, but is only distally related to other known members of the GNAT (GCN5-related $N$-acetyltransferase) family. Like SSAT1, SSAT2 is widely expressed, but did not turn over rapidly, and levels were unaffected by treatments with polyamine analogues. Despite similarity in sequence to SSAT1, polyamines were found to be poor substrates of purified SSAT2, having $K_{\mathrm{m}}$ values in the low millimolar range and $k_{\text {cat }}$ values of $<0.01 \mathrm{~s}^{-1}$. The $k_{\text {cat }} / K_{\mathrm{m}}$ values for spermine and spermidine for SSAT2 were $<0.0003 \%$ those of SSAT1. Expression of SSAT2 in NIH-3T3 cells was not
\end{abstract}

detrimental to growth, and did not reduce polyamine content or increase acetylpolyamines. These results indicate that SSAT2 is not a polyamine catabolic enzyme, and that polyamines are unlikely to be its natural intracellular substrates. A promising candidate for the physiological substrate of SSAT2 is thialysine [S-(2-aminoethyl)-L-cysteine], which is acetylated predominantly at the $\varepsilon$-amino group with $K_{\mathrm{m}}$ and $k_{\text {cat }}$ values of $290 \mu \mathrm{M}$ and $5.2 \mathrm{~s}^{-1}$. Thialysine is a naturally occurring modified amino acid that can undergo metabolism to form cyclic ketimine derivatives found in the brain and as urinary metabolites, which can undergo further reaction to form antioxidants. SSAT2 should be renamed 'thialysine $N^{\varepsilon}$-acetyltransferase', and may regulate this pathway.

Key words: acetylation, acetyl-CoA, polyamine, protein turnover, $S$-(2-aminoethyl)-L-cysteine (thialysine).

\section{INTRODUCTION}

Polyamines are essential regulators of mammalian cell growth and differentiation [1-3]. Intracellular polyamine concentrations are adjusted to cellular requirements by the concerted action of biosynthetic and catabolic enzymes in addition to transport mechanisms. Spermidine/spermine $N^{1}$-acetyltransferase (SSAT1) is a catabolic enzyme that plays a critical role in polyamine homoeostasis [4,5]. It contributes to the down-regulation of intracellular polyamine pools by catalysing the specific transfer of an acetyl group from acetyl-CoA to an aminopropyl group of spermine or spermidine. The $N^{1}$-acetyl derivatives formed may be excreted from the cell or are subject to further catabolism by acetylpolyamine oxidase, forming spermidine or putrescine respectively. Acetylation of the aminobutyl moiety of spermidine to form $N^{8}$-acetylspermidine also occurs physiologically, although the specific enzyme responsible for this reaction is not well defined. In general, acetylpolyamines do not persist in normal cells, although elevated levels of acetylpolyamines have been described in cancer cells [3] and may be associated with the progression of chemically induced skin tumours in transgenic mice that overexpress SSAT1 in the epidermis from a keratin-6 promoter [6].

SSAT1 is a short-lived enzyme whose activity is very low under basal cellular conditions. However, the levels of SSAT1 enzyme can be increased very readily in response to a number of physiological and pharmacological stimuli. Polyamine analogues such as BE-3-4-3 $\left[N^{1}, N^{12}\right.$-bis(ethyl)spermine $]$ and BE-3-3-3 $\left[N^{1}, N^{11}\right.$-bis(ethyl)norspermine], currently undergoing clinical evaluation as antitumour agents [7,8], are potent inducers of cellular SSAT1 activity. A component of the elevated activity produced by these agents involves inhibition of SSAT1 degradation by the $26 \mathrm{~S}$ proteasome, and we have previously described amino acid residues in SSAT1 that play an important role in conferring this rapid turnover $[9,10]$.

Studies by site-directed mutagenesis have provided some information identifying regions in the SSAT1 protein that are important for such properties as catalytic activity, substrate binding and stability of the enzyme [9-14]. Little additional information of key residues can be obtained by comparing SSAT1 sequences from different species, since these proteins are virtually identical. We have therefore attempted to identify other more distally related acetyltransferases that would facilitate the process of assigning properties to specific regions of the protein based on primary amino acid sequence similarity. To this end, we identified a related sequence in Schizosaccharomyces pombe using BLAST (basic local alignment search tool) homology searches as well as a human homologue that we originally termed SSAT2 [15] without reference to its functional properties. A recent paper also described SSAT2, and suggested that it may be compartmentalized in the cell, since it did not appear to influence

Abbreviations used: AECK, aminoethyl-L-cysteine ketimine; AECK-DD, decarboxylated dimer of AECK; BE-3-3-3, $N^{1}, N^{11}$-bis(ethyl)norspermine BE-3-4-3, $N^{1}, N^{12}$-bis(ethyl)spermine; BLAST, Basic Local Alignment Search Tool; DTNB, 5,5'-dithiobis-(2-nitrobenzoate); ESI, electrospray ionization; EST, expressed sequence tag; GNAT, GCN5-related $N$-acetyltransferase; IPTG, isopropyl $\beta$-D-thiogalactoside; MALDI-TOF-MS, matrix-assisted laserdesorption ionization-time-of-flight mass spectrometry; OPA, o-phthaldialdehyde; RT-PCR, reverse-transcriptase-PCR; SSAT, spermidine/spermine- $N^{1}$. acetyltransferase; TMA, 1,4-thiomorpholine-3.5-dicarboxylic acid.

1 To whom correspondence should be addressed (email csc6@psu.edu). 
polyamine levels in transiently transfected HEK-293 cells [16].

In the present study, we have studied the properties of human SSAT2 in detail using purified recombinant protein, studies of the turnover of the protein by the ubiquitin/proteasome system in reticulocyte lysates, and studies of cells stably transfected with a construct expressing SSAT2. The $S$. pombe SSAT2 was also studied using purified recombinant protein. Our findings show that, in contrast with SSAT1, polyamines are poor substrates for SSAT2 and that SSAT2, although widely expressed, is unlikely to influence cellular polyamine content. A probable physiological substrate is thialysine [ $S$-(2-aminoethyl)-L-cysteine], which is very efficiently acetylated on the $\varepsilon$-amino group to form $S$-(2acetylaminoethyl)-L-cysteine. SSAT2 does, however, provide a useful structural comparison with SSAT1, since it does not turn over rapidly or respond to polyamine analogues, and binds polyamine substrates very weakly.

\section{MATERIALS AND METHODS}

\section{Materials}

All reagents were purchased from Fisher Scientific (Pittsburgh, PA, U.S.A.) or Sigma Chemical Co. (St Louis, MO, U.S.A.), unless stated otherwise. Oligodeoxynucleotides were synthesized in the Macromolecular Core Facility, Hershey Medical Center, PA, U.S.A. Restriction enzymes were from New England Biolabs, Inc. (Beverly, MA, U.S.A.) and Roche Molecular Biochemicals (Indianapolis, IN, U.S.A.). L- $\left[{ }^{35} \mathrm{~S}\right]$ Methionine and L$\left[{ }^{35} \mathrm{~S}\right]$ cysteine $/\left[{ }^{35} \mathrm{~S}\right]$ methionine (both translation grade; $1000 \mathrm{Ci} /$ mmol were obtained from DuPont NEN, Boston, MA, U.S.A.). $\left[1-{ }^{14} \mathrm{C}\right]$ acetyl-CoA $(50 \mathrm{Ci} / \mathrm{mol})$ was purchased from ICN Biochemicals (Costa Mesa, CA, U.S.A.). TNT T7-coupled rabbit reticulocyte lysate translation system and RNasin were from Promega (Madison, WI, U.S.A.). Anti-hSSAT2 antibody was derived from rabbits immunized with purified human SSAT2 protein at Cocalico Biologicals (Reamstown, PA, U.S.A.). Plasmid purification columns were from Qiagen Inc. (Valencia, CA, U.S.A.). BE-3-4-3 and BE-3-3-3 were kindly provided by Dr Raymond J. Bergeron (University of Florida, Gainesville, FL, U.S.A.). Multiple-tissue cDNA (MTC ${ }^{\mathrm{TM}}$ ) Panels and AdvanTaq Plus $^{\mathrm{TM}}$ DNA polymerase were purchased from Clontech (Palo Alto, CA, U.S.A.). Transfection reagents: LIPOFECTAMINE ${ }^{\mathrm{TM}}$ Plus was from Invitrogen Life Technologies (Carlsbad, CA, U.S.A.). G418 sulphate was from Cellgro (Herndon, VA, U.S.A.). Reagents for Western blotting included PVDF membrane (Millipore Corp, Bedford, MA, U.S.A.) and the Phototop ${ }^{\mathrm{TM}}$-Horse Radish Peroxidase chemiluminescent detection system from Cell Signaling Technology Inc. (Beverly, MA, U.S.A.). Protein concentration was determined by the Bradford method using BSA (fraction V) as a standard [17].

\section{Cloning of human SSAT2 and plasmid construction}

All of the listed constructs were made by PCR. Forward (5'GGGGATCCATATGGCTTCCGTGCGGATC-3') and reverse (5'-AGTACTATCGATTCACTTTCCTGCCAACTTTCT-3') oligodeoxynucleotides with unique $N d e 1$ and $C l a 1$ restriction sites were designed to flank the human SSAT2 cDNA sequence. PCR products of the anticipated size $(534 \mathrm{bp})$ were amplified from human fetal liver and HeLa cell cDNA libraries (Stratagene, La Jolla, CA, U.S.A.). The product from the HeLa cell library was purified and digested with $N d e 1$ and Cla 1 for cloning into the same restriction sites of pT7 plasmids with or without an encoded $\mathrm{N}$-terminal $\mathrm{His}_{6}$ epitope tag. The sequence of the pT7-
hSSAT2 clones was verified in the Macromolecular Core Facility at Hershey Medical Center.

A clone for the $S$. pombe SSAT2 (described under the accession number AAB39945 in the GenBank ${ }^{\circledR}$ database as the atsl gene product) was kindly provided by Dr Martin Lutzelberger and Dr Norbert F. Kaufer, Technical University, Braunschweig, Germany. The ats1 cDNA in the pUC plasmid was digested with BamH1 and cloned into the same sites of the pBluescript cloning vector (Stratagene, La Jolla, CA, U.S.A.) for in vitro translation reactions, and into the $\mathrm{pQE} 30$ bacterial expression plasmid (Qiagen Inc.).

\section{Expression of SSAT1 and SSAT2 mRNA in human tissues}

Human Multiple Tissue cDNA (MTC ${ }^{\mathrm{TM}}$ ) Panels I and II are sets of normalized, first-strand cDNA generated by RT-PCR (reverse transcriptase-PCR) using poly (A) ${ }^{+}$(polyadenylated) RNA from different human tissues (Clontech). These cDNAs were used as templates for PCR to examine the expression profile of SSAT2 and SSAT1 in 16 different tissues. Forward PCR primers with the sequence 5'-ATTCTTCCAGCGCCCGGGAAGCTACTG-3' for SSAT2 and 5'-GTGCCGAAAGAGCACTGGACTCCGGAA$3^{\prime}$ for SSAT1 were designed to anneal to the complementary cDNA strand that encodes amino acids 59-67 within each coding region. Reverse primers with sequence 5'-CTCAAGACAGAGATCCTCCTAGGGATG-3' for SSAT2 and 5'-GGAGGTTGTCATCTACAGCAGCACTCC-3' for SSAT1 were designed to anneal to a region within the respective $3^{\prime}$-UTR sequences. PCR products of the predicted size (369 bp) were generated for both SSAT2 and SSAT1 using the 'hot-start' PCR conditions recommended by Clontech. Control reactions were run in parallel and contained template and primers to amplify a $1 \mathrm{~kb}$ fragment of the housekeeping gene glyceraldehyde-3-phosphate dehydrogenase. PCR products were resolved on a $2 \%$ agarose/ethidium bromide gel.

\section{Expression and purification of recombinant proteins in Escherichia coli}

The pT7-hSSAT2 plasmid with an encoded N-terminal $\mathrm{His}_{6}$ epitope was expressed in BL21(DE3) lysogen E. coli (Novagen, Madison, WI, U.S.A). Bacterial cultures were grown in LuriaBertani medium supplemented with $50 \mu \mathrm{g} / \mathrm{ml}$ ampicillin to a $D_{600}$ of $\approx 0.5$, and grown for an additional $3 \mathrm{~h}$ following induction with $1 \mathrm{mM}$ IPTG (isopropyl $\beta$-D-thiogalactoside). Bacterial cell pellets were resuspended in $25 \mathrm{mM}$ Tris/ $\mathrm{HCl}, \mathrm{pH} 8.0$, lysed by sonication and centrifuged at $60000 \mathrm{~g}_{\text {av }}$ for $60 \mathrm{~min}$. The supernatant was applied to a TALON ${ }^{\mathrm{TM}}$ metal-affinity resin from Clontech, and the $\mathrm{His}_{6}$-tagged SSAT2 protein was eluted using an optimized imidazole gradient. Fractions that appeared as a single band on a denaturing $8 \%$ Tricine gel were pooled and dialysed extensively against $25 \mathrm{mM}$ Tris/ $\mathrm{HCl}, \mathrm{pH} 8.0$, containing $1 \mathrm{mM}$ dithiothreitol and concentrated using an Amicon Centricon-10 microconcentrator (Millipore). Both the human SSAT1 and $S$. pombe SSAT proteins were expressed from the pQE30 plasmid in XL1-Blue E. coli, and purified as described previously [14].

\section{Assay of acetyltransferase activity}

Purified SSAT2 protein was assayed for polyamine acetyltransferase activity at $30^{\circ} \mathrm{C}$ using the method of binding to cellulose phosphate discs to separate the ${ }^{14} \mathrm{C}$-acetylated product from the $\left[1-{ }^{14} \mathrm{C}\right]$ acetyl-CoA substrate [18]. A standard assay mixture contained $50 \mathrm{mM}$ Tris/ $\mathrm{HCl}, \mathrm{pH} 8.0,3 \mathrm{mM}$ polyamine substrate (unless stated otherwise) and $16 \mu \mathrm{M}\left[1-{ }^{14} \mathrm{C}\right]$ acetyl-CoA in a total 
volume of $100 \mu \mathrm{l}$. This assay relies on the ability of the positively charged acetylated product to bind to cellulose phosphate, and cannot be used for potential substrates that form neutral or acidic products.

A spectrophotometric assay that relies on measuring the generation from acetyl-CoA of free CoA that reacts with DTNB [5,5'-dithiobis-(2-nitrobenzoate)] was used to determine the ability of thialysine to act as an acetyl acceptor [19]. Unless stated otherwise, the standard assay mixture contained $100 \mathrm{mM}$ Tris/ $\mathrm{HCl}, \mathrm{pH} 8.0,1 \mathrm{mM}$ acetyl-CoA and $10 \mathrm{mM}$ thialysine that was linear for up to $20 \mathrm{~min}$ when incubated at $30^{\circ} \mathrm{C}$ using $100 \mathrm{ng}$ of purified human or $50 \mathrm{ng}$ of $S$. pombe SSAT2. Reactions were terminated by addition of $150 \mu \mathrm{l}$ of ethanol, after which was added $0.5 \mathrm{ml}$ of a $0.2 \mathrm{mM}$ solution of DTNB dissolved in $100 \mathrm{mM}$ Tris/ $\mathrm{HCl}, \mathrm{pH}$ 8.0. Samples were centrifuged at $16000 \boldsymbol{g}_{\mathrm{av}}$ for $5 \mathrm{~min}$, and the supernatant was used to determine the absorbance at $412 \mathrm{~nm}$ measured against a blank that lacked thialysine. The stoichiometric amount of 5-thio-2-nitrobenzoic acid generated from DTNB by reduction with CoASH released by the enzyme reaction was determined using a molar absorption coefficient of $14140 \mathrm{M}^{-1} \cdot \mathrm{cm}^{-1}$ [19]. Purified SSAT2 from both human and $S$. pombe were assayed for their ability to use thialysine as a substrate.

\section{HPLC analysis}

Polyamines were determined by reversed-phase HPLC using post-column derivatization with OPA (o-phthaldialdehyde) and fluorescence detection, as described previously [20]. Alternatively, the HPLC column was linked directly to a Canberra Packard Flow-one $\beta$-radiochemical detector for identification of $\left[{ }^{14} \mathrm{C}\right]$ acetyl-polyamine products of the acetyltransferase assays using Flo-Scint II scintillation cocktail (Packard Bioscience, Meridan, CT, U.S.A.) [21].

The following HPLC method was used to analyse thialysine and its $\alpha$-and $\varepsilon$-acetyl-derivatives. Standards or aliquots of deproteinized enzyme assays were derivatized with equal volumes of OPA reagent $[40 \mathrm{mM}$ OPA/200 $\mathrm{mM}$ potassium phosphate buffer (pH 9.4)/0.14 mM 2-mercaptoethanol/10\% methanol] and subjected to a Spherisorb ODS II $(5 \mu \mathrm{M}, 125 \mathrm{~mm} \times 3 \mathrm{~mm})$ reversed-phase HPLC. The mobile phase consisted of solvent A (10 mM potassium dihydrogen phosphate, $\mathrm{pH}$ 5.9) and solvent B (acetonitrile/methanol/water; 4:3:3, by vol.). Separation was performed at a flow rate of $0.9 \mathrm{ml} \cdot \mathrm{min}^{-1}$ under application of the following gradient (percentages of solvent $\mathrm{B}$ are shown): $0 \mathrm{~min}, 20 \%$; $4 \mathrm{~min}, 27 \%$; $11.5 \mathrm{~min}, 27 \%$; $28 \mathrm{~min}, 100 \%$; $31 \mathrm{~min}, 100 \%$; $40 \mathrm{~min}, 20 \%$; $58 \mathrm{~min}, 20 \%$. OPA derivatives were detected with a fluorescence spectrophotometer (excitation wavelength $338 \mathrm{~nm}$, emission wavelength $425 \mathrm{~nm}$, SFM 25, Kontron, Neufahrn, Germany) and by a flow-through radiodetector (LB 506; Berthold, Pforzheim, Germany). The retention times of $\alpha$ - and $\varepsilon-N$-acetyl-thialysine were $9.52 \mathrm{~min}$ and $6.77 \mathrm{~min}$ respectively.

\section{Mass spectrometry}

Samples for analysis by MALDI-TOF-MS (matrix-assisted laserdesorption ionization-time-of-flight mass spectrometry) were mixed 1:1 with matrix solution containing $10 \mathrm{mg} / \mathrm{ml}$ dihydrobenozoic acid (Sigma) in $30 \%$ (v/v) acetonitrile/0.1\% trifluoroacetic acid. After mixing each sample with the matrix, $0.8 \mu \mathrm{l}$ of the mixture was spotted on to a stainless-steel MALDI target plate and allowed to air-dry, with control spots containing matrix alone spotted in contiguous spots. MALDI-TOF spectra in the range of $m / z 10-1000$ were acquired on an Applied Biosystems
4700 Proteomics Analyzer in positive-ion reflectron mode, using a laser power setting of 2900 .

ESI (electrospray ionization) mass spectra were obtained in the positive-ion mode using a Quattro II mass spectrometer (Micromass, Beverly, MA, U.S.A.). Aliquots $(20 \mu \mathrm{l})$ of each solution were mixed with $20 \mu$ l of HPLC-grade acetonitrile (Fisher Scientific), and $10 \mu \mathrm{l}$ aliquots were introduced to the mass spectrometer by flow injection with $100 \mu \mathrm{l} / \mathrm{min}$ flow of acetonitrile/water $(50: 50, v / v)$. Full-scan spectra were obtained over the range of $\mathrm{m} / \mathrm{z} 50-500$ at $2 \mathrm{~s} / \mathrm{scan}$. Product ion MS/MS spectra were obtained using identical ionization conditions, except that $1.8 \times 10^{-3}$ mbar of argon was used as the collision gas, with a collision cell potential of $-20 \mathrm{~V}$.

\section{Construction of pLNCX-FLAG-SSAT2 and expression of SSAT2 in NIH3T3 cells}

The human SSAT2 cDNA with an N-terminal FLAG epitope (encoding MDYKDDDDK) was cloned into the HindIII-ClaI restriction sites of the retroviral pLNCX mammalian expression vector (Clontech) using the following strategy: the pT7-His SSAT2 plasmid was digested with NdeI and BamHI to remove $15 \mathrm{bp}$ of N-terminal sequence, and the desired large fragment was gel-purified. The N-terminal FLAG epitope was generated after annealing forward (5'-TAAAGCTTACCATGGATTACAAGGATGACGACGATAAGATGGCTTCCGTGCG-3') and reverse (5'-GATCCGCACGGAAGCCATCTTATCGTCGTCATCCTTGTAATCCATGGTAAGCTT-3') primers that encoded HindIII and BamHI restriction sites at the $5^{\prime}$ and $3^{\prime}$ ends respectively.

The annealed primers were ligated to the BamHI overhang of the NdeI-BamHI vector fragment to generate a linear template for PCR. The desired HindIII-ClaI FLAG-SSAT2 PCR product was generated using the above-mentioned forward primer and a reverse primer (5'-AGTACTATCGATTCACTTTCCTGCCAACTTTCT- $\left.3^{\prime}\right)$ that encoded a $C l a I$ restriction site downstream of the stop codon for SSAT2. The PCR product was digested with HindIII and ClaI, and ligated into the same sites of a mammalian expression vector to generate the construct pLNCXFLAG-SSAT2.

NIH3T3 cells were seeded at $2 \times 10^{5}$ cells $/ 60 \mathrm{~mm}$ dish and grown overnight in Dulbecco's modified Eagle's medium supplemented with $2 \mathrm{mM}$ L-glutamine, $10 \%$ fetal-calf serum and 100 units $/ \mathrm{ml}$ penicillin $/ 100 \mu \mathrm{g} / \mathrm{ml}$ streptomycin. Cells were transfected with $2 \mu \mathrm{g}$ of pLNCX-FLAG-SSAT2 plasmid for $5 \mathrm{~h}$ at $37{ }^{\circ} \mathrm{C}$ in a humidified atmosphere of $5 \% \mathrm{CO}_{2}$ using the LIPOFECTAMINE Plus ${ }^{\mathrm{TM}}$ reagent, as described by the manufacturer (Invitrogen). The medium was changed $5 \mathrm{~h}$ after the start of transfection, and the cells were left to grow for an additional $48 \mathrm{~h}$ before harvesting. Cells were re-plated after this time at $5 \times 10^{4}, 10 \times 10^{4}$ and $20 \times 10^{4}$ cells $/ 100 \mathrm{~mm}$ dish in the presence of $0.5 \mathrm{mg} / \mathrm{ml} \mathrm{G} 418$ selection medium, and maintained until resistant colonies were established. Individual G418-resistant colonies were selected by trypsin treatment using cloning cylinders, after which they were transferred to 24 -well plates and allowed to expand.

\section{Degradation assay}

The degradation of $\left[{ }^{35} \mathrm{~S}\right]$ cysteine $/\left[{ }^{35} \mathrm{~S}\right]$ methionine-labelled SSAT2 and $\left[{ }^{35} \mathrm{~S}\right]$ methionine-labelled SSAT1, generated as non-histidinetagged proteins from pT7 and p9.3 plasmids respectively in a coupled transcription/translation TNT assay (Promega), was studied using a rabbit reticulocyte-based degradation assay, as described previously $[9,10]$. 


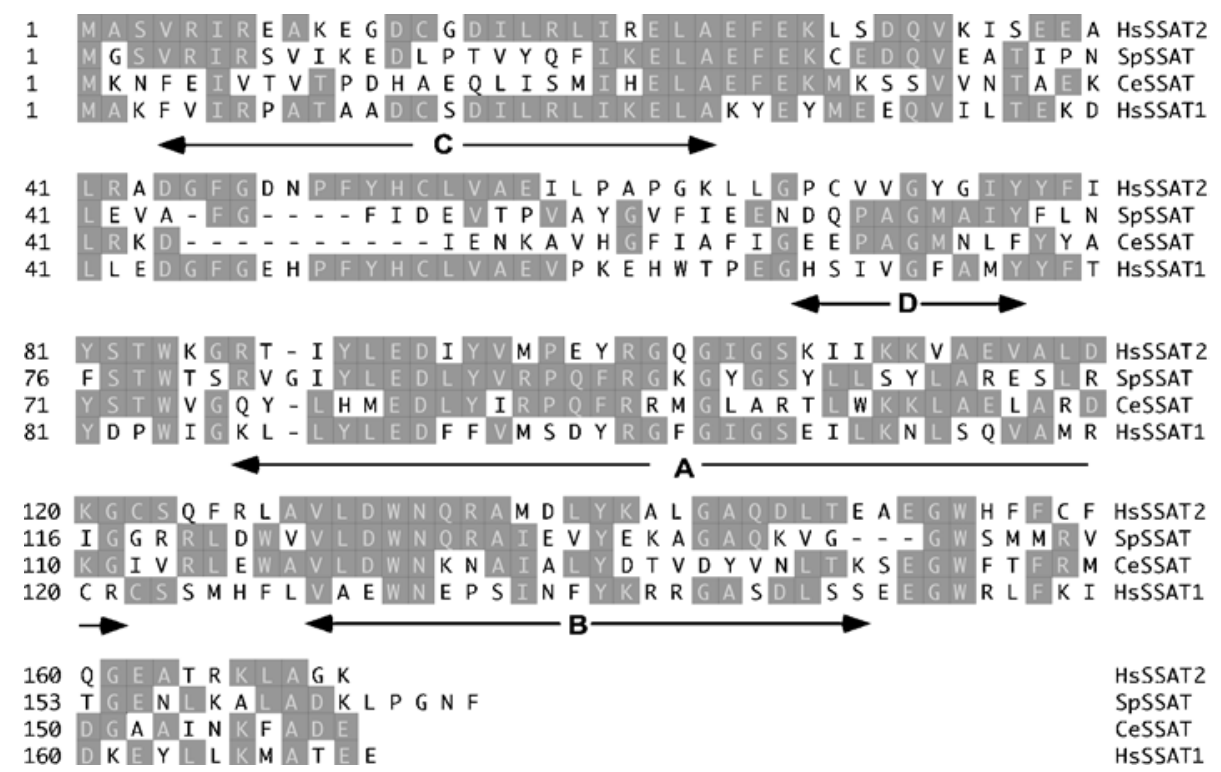

Figure 1 Alignment of $\mathrm{N}$-acetyltransferase homologues

Protein sequences were aligned using the ClustalW program. Human SSAT1(hSSSAT1) is compared with human SSAT2 (hsSSAT2), the S. pombe ats1 gene product designated SpSSAT (accession no. U82218) and the C. elegans protein designated CeSSAT (accession no. NP_505978). Arrows indicate motifs discussed further in the text. Conserved amino acids are shaded.

\section{RESULTS}

\section{Identification of a human cDNA encoding a novel acetyltransferase (SSAT2)}

We originally identified a novel human acetyltransferase (accession no. AAL83905) as SSAT2 [15], on the basis of sequence similarity to human SSAT1, without reference to the functional properties of this protein. SSAT2 was discovered using the human SSAT1 protein sequence to conduct a tBLASTn search of several eukaryotic EST (expressed sequence tag) databases. Several overlapping human sequences were identified and spliced together to form one contiguous sequence. The entire contiguous human sequence that comprised six exons with well-defined exon-intron boundaries (accession number AC008049) was found to be present in the high-throughput genomic sequence (HTGS) database, giving this gene a chromosomal location of 17p13.1, whereas human SSAT1 is localized to Xp22.1 [22]. Similar results have been published recently by Chen et al. [16].

\section{Primary amino acid sequence comparison of acetyltransferases that have homology with human SSAT1}

The human SSAT2 cDNA encodes a 170-amino-acid protein that is shown in Figure 1 in alignment with similar sequences from $S$. pombe and $C$. elegans for comparison with human SSAT1 ('HsSSAT1' in the Figure). The human SSAT2 protein ('HsSSAT2' in the Figure) contains the highly conserved acetyl-CoAbinding domain identified previously in SSAT1 as RGXGIGS [13], part of the universally conserved motif $\mathrm{A}$ in the GNAT superfamily [23], which includes $\operatorname{Arg}^{101}$ known to be essential for catalytic activity $[12,13]$. All of the enzymes shown have highly conserved residues found in human SSAT1 that forms part of motif D and motif B respectively in the GNAT superfamily. There is only weak similarity to motif $C$ present in GNAT family members. Of the enzymes shown, human SSAT2 is closest in sequence similarity to human SSAT1 showing $46 \%$ identity and $61 \%$ homology with human SSAT1. The identity/homology values for $S$. pombe SSAT2 of $29 \% / 43 \%$ and for $C$. elegans
SSAT2 of $26 \% / 45 \%$ are compared with human SSAT1. $S$. pombe SSAT2 and C. elegans SSAT2 are $38 \% / 49 \%$ and $34 \% / 49 \%$ identical/similar respectively when compared with the human SSAT2 sequence, making them closer in sequence to SSAT2 than to SSAT1. Notably, both the human SSAT2 and $S$. pombe SSAT2 sequences lack the two acidic residues present in the previously described C-terminal MATEE sequence motif of SSAT1 that was shown to be important in the rapid degradation of SSAT1 in vitro [9].

\section{SSAT2 expression in human tissues}

The expression profile of SSAT2 and SSAT1 mRNA was examined in a wide selection of human tissues using commercially available cDNA (generated by RT-PCR in the form of MTC ${ }^{\mathrm{TM}}$ panels) as a template for the PCR reaction. Both SSAT1 and SSAT2 were found to be widely expressed, as judged by a band corresponding to the predicted $369 \mathrm{bp}$ product that was amplified in all 16 tissues examined (Figures $2 \mathrm{~A}$ and $2 \mathrm{~B}$ ). The expression of SSAT2 mRNA was comparable with that of SSAT1 in all tissues tested. In the case of SSAT2, a smaller PCR product was also detected (Figure 2A) that may represent the amplification of an alternatively spliced form of the SSAT2 gene.

\section{Kinetic properties of human SSAT2 and S. pombe SSAT2 purified from $E$. coli}

The human SSAT1 and the human and $S$. pombe SSAT2 proteins, all with (His) ${ }_{6}$ tags, were expressed in bacteria from the pT7 or pQE30 constructs described in the Materials and methods section and a gel showing purity and mobility of the purified proteins is shown in Figure 3B (lanes 3-5). (The predicted mass of each protein with epitope is $21.2 \mathrm{kDa}, 20.1 \mathrm{kDa}$ and $20.5 \mathrm{kDa}$ for human SSAT1, human SSAT2 and $S$. pombe SSAT2 respectively). Although SSAT1 and SSAT2 are similar in size, there was a difference observed in their mobility when resolved on an SDS/15\%-PAGE gel. In particular, the human SSAT2 protein showed a faster-than-predicted mobility under denaturing 

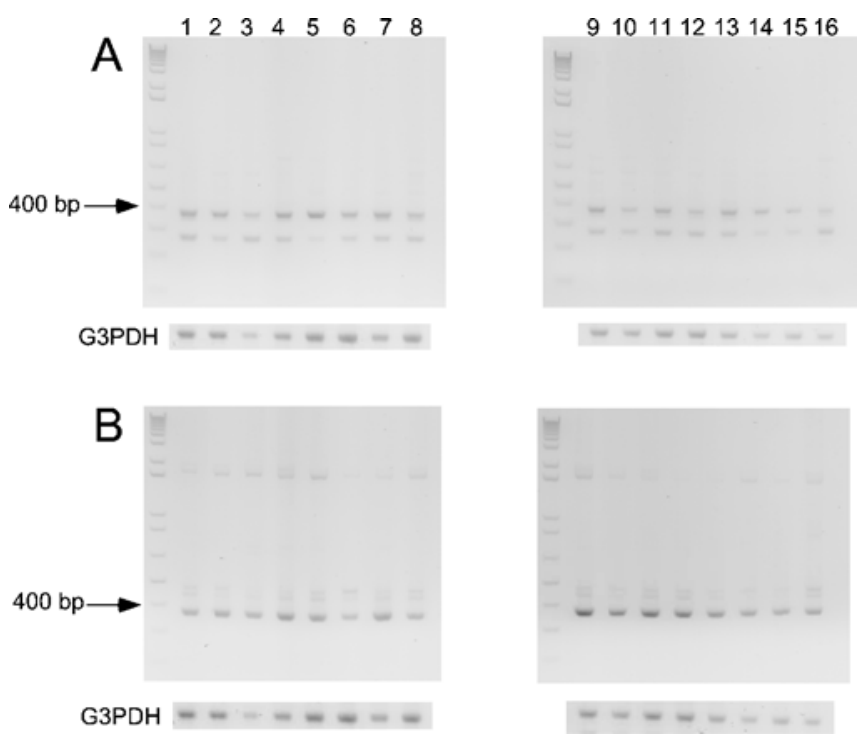

Figure 2 Comparison of the tissue expression pattern of human SSAT2 and SSAT1 mRNA in multiple human tissues

MTC ${ }^{\text {TM }}$ Panels I and II containing CDNAs derived by RT-PCR from the listed human tissue sources were used as templates for PCR to screen for expression of transcripts corresponding to SSAT2 (A) and SSAT1 (B). Primers were designed to amplify 369 bp products as described in the Materials and methods section. Lanes 1-16 are identical for each panel, and correspond to the following tissues: lane 1, heart; lane 2 brain; lane 3, placenta; lane 4, lung; lane 5, liver; lane 6, skeletal muscle; lane 7, kidney; lane 8, pancreas; lane 9, spleen; lane 10, thymus; lane 11, prostate; lane 12, testis; lane 13, ovary; lane 14, small intestine; lane 15, colon; and lane 16, peripheral blood leukocyte. G3PDH, glyceraldehyde-3-phosphate control reactions.

conditions. In order to check that the purified protein was full length, the molecular mass of the human SSAT2 protein was confirmed to be $20.1 \mathrm{kDa}$ by MALDI-TOF-MS (results not shown).

It should also be noted that the human SSAT2 and $S$. pombe SSAT2 proteins ran with similar characteristics with respect to human SSAT1 in SDS/PAGE when expressed as ${ }^{35} \mathrm{~S}$-labelled proteins from the pBluescript plasmid (results not shown). This suggests that the altered mobilities observed using SDS/PAGE are due to the unique composition of each protein.

The purified enzymes shown in Figure 3 were examined for their substrate specificity using the polyamines listed in Table 1 as putative acetyl acceptors. The polyamines were found to be poor acetyl acceptors in the reactions brought about by SSAT2 from human or $S$. pombe sources. The $K_{\mathrm{m}}$ values were in the millimolar range $(1.5-15 \mathrm{mM})$ and the $k_{\text {cat }}$ values were very low $\left(<0.025 \mathrm{~s}^{-1}\right)$. These results contrast strikingly with the known properties of SSAT1, which has $K_{\mathrm{m}}$ values for spermidine and spermine in the 4-60 $\mu \mathrm{M}$ range [18] and $k_{\text {cat }}$ values of 5$8 \mathrm{~s}^{-1}$. For example, with spermine and spermidine as substrates for human SSAT2, the $k_{\mathrm{cat}} / K_{\mathrm{m}}$ was $<0.6 \mathrm{M}^{-1} \cdot \mathrm{s}^{-1}$, whereas with human SSAT1, the $k_{\text {cat }} / K_{\mathrm{m}}$ values are $150000 \mathrm{M}^{-1} \cdot \mathrm{s}^{-1}$ for spermidine and $1351350 \mathrm{M}^{-1} \cdot \mathrm{s}^{-1}$ for spermine [14]. Of the polyamines tested, the best diamine substrate for human SSAT2 was actually 1,3-diaminopropane, with a $k_{\text {cat }} / K_{\mathrm{m}}$ value of $8.7 \mathrm{M}^{-1}$. $\mathrm{s}^{-1}$. This is a poor substrate for SSAT1, with a $k_{\text {cat }} / K_{\mathrm{m}}$ of $3.1 \mathrm{M}^{-1} \cdot \mathrm{s}^{-1}[18]$.

The predominant product of the action of SSAT2 on spermidine was found to be $N^{1}$-acetylspermidine after separation of the products by HPLC. This is consistent with the preference for 1,3-diaminopropane over putrescine (1,4-diaminobutane) as a substrate and also with the finding that $N^{8}$-acetylspermidine,
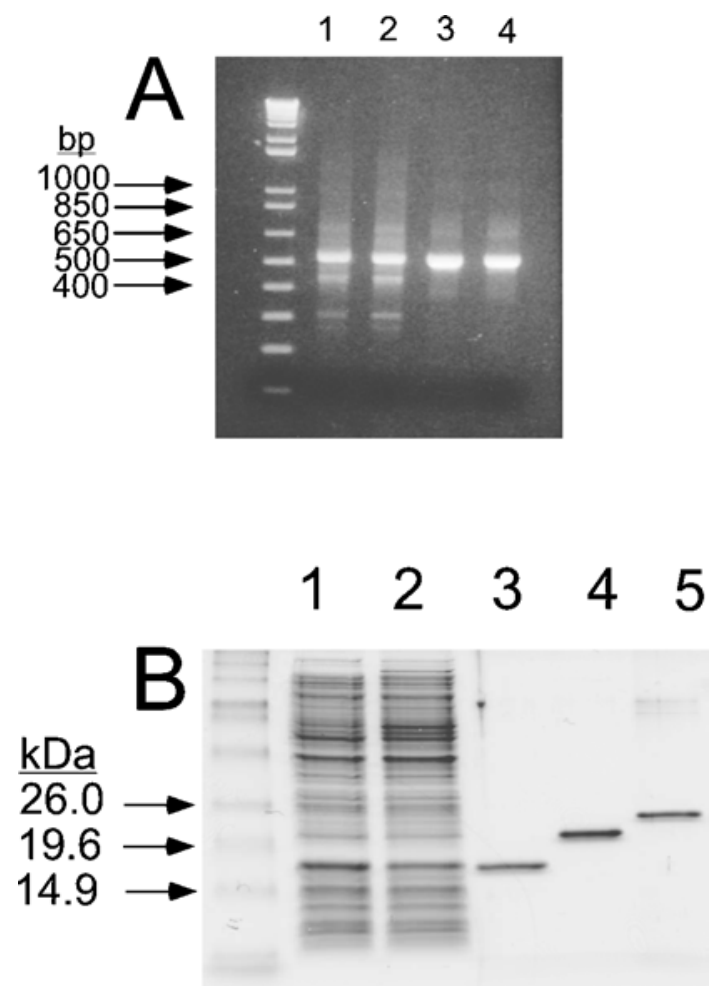

Figure 3 Cloning of human SSAT2 and expression of human and S. pombe SSAT2 in E. coli

The hsSSAT2 CDNA was cloned by PCR as described in the Materials and methods section, and the reaction products were resolved by $2 \%$ agarose gel electrophoresis. (A) shows PCR products (in duplicate) amplified from a human fetal liver library (lanes 1 and 2) and a HeLa cell library (lanes 3 and 4). (B) shows purified histidine-tagged proteins resolved on an SDS/15\% polyacrylamide gel. Lanes 1 and 2 are, respectively, the crude soluble E. coli extract before and after induction by IPTG. Lane 3 shows purified $\mathrm{His}_{6}$-tagged human SSAT2 expressed from the pT7-7 plasmid in the BL21(DE3) E. coli strain, Lanes 4 and 5 are, respectively, purified $\mathrm{His}_{6}$-tagged human SSAT1 and $\mathrm{His}_{6}$-tagged S. pombe SSAT2, both expressed from the pQE30 plasmid in XL1-Blue cells.

but not $N^{1}$-acetylspermidine, formed a product with a predicted retention time corresponding to the diacetylspermidine derivative (results not shown).

Many other compounds were tested as putative substrates for SSAT2, but were not active, including L-canavanine and taurine (results not shown). It was found that thialysine was a much better substrate than polyamines. Thus, with human SSAT2, the $k_{\text {cat }} / K_{\mathrm{m}}$ was $18100 \mathrm{M}^{-1} \cdot \mathrm{s}^{-1}$, and for $S$. pombe SSAT2 it was $3600 \mathrm{M}^{-1} \cdot \mathrm{s}^{-1}$. Thialysine was also shown to inhibit the acetylation of spermine with an $\mathrm{ED}_{50}$ value of $7.4 \mu \mathrm{M}$ in an assay containing purified human SSAT2 at a fixed spermine concentration of $3 \mathrm{mM}$, confirming a preference for thialysine over spermine for the enzyme. Human SSAT2 did acetylate L-lysine, but less efficiently than thialysine $(\approx 20 \%$ of the specific activity using $500 \mathrm{ng}$ of purified enzyme, $1 \mathrm{mM}$ acetyl-CoA with $10 \mathrm{mM}$ L-lysine substituted for thialysine). Although the S. pombe SSAT2 had a relatively poor affinity for thialysine with a $K_{\mathrm{m}}$ of $12.7 \mathrm{mM}$ compared with $0.29 \mathrm{mM}$ for human SSAT2, it did have a high turnover number of approx. $46 \mathrm{~s}^{-1}$ compared with the hSSAT2 enzyme for this substrate. There was no reaction of SSAT1 with thialysine, even in assays containing $1 \mu \mathrm{g}$ of the hSSAT1, $10 \mathrm{mM}$ thialysine and $1 \mathrm{mM}$ acetyl-CoA. These results show a clear difference in substrate specificity for the respective purified enzymes. 
Table 1 Kinetic analysis of purified recombinant human and $S$. pombe SSAT2

Apparent $K_{\mathrm{m}}$ and $k_{\text {cat }}$ values were determined for the polyamine substrates using the filter binding assay described in the Materials and methods section at a fixed concentration of $16 \mu \mathrm{M}$ $\left[{ }^{14} \mathrm{C}\right]$ acetyl-CoA, whereas the polyamine substrate concentration was varied from $0.05 \mathrm{mM}$ up to $20 \mathrm{mM}$. Kinetic parameters shown using thialysine as an acetyl acceptor were determined in duplicate experiments using the spectrophotometric assay described in the Materials and methods section over a concentration range of $0.05-2 \mathrm{mM}$ using $100 \mathrm{ng}$ of human SSAT2 in the assay and from $0.05-8 \mathrm{mM}$ using $50 \mathrm{ng}$ of $S$. pombe SSAT2 in the presence of $1 \mathrm{mM}$ acetyl-CoA. (Values agreed within a $5 \%$ error).

\begin{tabular}{|c|c|c|c|c|}
\hline Source & Substrate & $K_{\mathrm{m}}(\mathrm{mM})$ & $k_{\text {cat }}\left(\mathrm{S}^{-1}\right)$ & $k_{\text {cat }} / K_{\mathrm{m}}\left(\mathrm{M}^{-1} \cdot \mathrm{S}^{-1}\right)$ \\
\hline \multirow[t]{6}{*}{ Human SSAT2 } & Putrescine* & 8.2 & 0.0023 & 0.28 \\
\hline & Spermidine $\dagger$ & 13.4 & 0.0072 & 0.54 \\
\hline & Spermine $\dagger$ & 4.8 & 0.0020 & 0.42 \\
\hline & 1,3-Diaminopropane* & 1.6 & 0.0137 & 8.7 \\
\hline & Norspermidine* & 6.0 & 0.0168 & 2.8 \\
\hline & Thialysine & 0.29 & 5.25 & 18100 \\
\hline \multirow[t]{4}{*}{ S. pombe SSAT2 } & Putrescine* $^{\star}$ & 4.3 & 0.0117 & 2.7 \\
\hline & Spermidine $\dagger$ & 4.6 & 0.0243 & 5.3 \\
\hline & Spermine $\dagger$ & 1.8 & 0.0167 & 9.3 \\
\hline & Thialysine & 12.7 & 46 & 3600 \\
\hline \multirow[t]{3}{*}{ Human SSAT1 } & Spermidine:: & 0.058 & 8.7 & 150000 \\
\hline & Spermineł & 0.0037 & 5 & 1351350 \\
\hline & Thialysine & No reaction & & \\
\hline
\end{tabular}

Each value shown was determined in single ${ }^{*}$ or duplicate $\uparrow$ experiments (variation up to $28 \%)$.

¥Values shown for human SSAT1 are published data using the filter binding assay [14].

\section{Identification of $\boldsymbol{N}^{\varepsilon}$-acetylthialysine as the product formed using thialysine as a substrate}

Since thialysine has two primary amine groups that can be acetylated, two approaches (MS and HPLC) were used to determine the product of the reaction. MALDI-TOF spectra of thialysine mixed with dihydrobenzoic acid showed the expected $(M+\mathrm{H})^{+}$peak of thialysine at 165 . After reaction with acetylCoA and SSAT2, the resulting mass spectra showed peaks at both $\mathrm{m} / \mathrm{z} 165$ and 207, consistent with acetylation of thialysine. Spectra from control incubations in which thialysine was not included (SSAT2 and acetyl-CoA only) did not show peaks at either $\mathrm{m} / \mathrm{z}$ 165 or 207, indicating that the $m / z 207$ peak observed was specific to acetylation of thialysine. A much smaller peak of $\mathrm{m} / \mathrm{z} 207$ was also observed in control incubations lacking enzyme (results not shown). Since non-enzymic acetylation of primary amines by acetyl-CoA occurs quite readily, this probably represents such non-enzymic acetylation of the thialysine occurring during the $1 \mathrm{~h}$ incubation with $1 \mathrm{mM}$ acetyl-CoA.

MS/MS fragmentation of the acetylated thialysine at $\mathrm{m} / \mathrm{z} 207$ was performed using matrix-free ESI on a Quattro II triplequadrupole mass spectrometer to determine whether the SSAT2induced acetylation of thialysine was occurring on the $\alpha$ - or on the $\varepsilon$-amine moiety. As seen in Figure 4(A), the full-scan MS spectrum obtained on this instrument showed the same thialysine and acetylated thialysine peaks $(\mathrm{m} / \mathrm{z} 165$ and 207) as observed with the MALDI instrument. Typical collision-induced fragmentation spectra produced from the acetylated thialysine peak at $\mathrm{m} / \mathrm{z} 207$ are shown in Figure 4(B). The major fragment ions present in the MS/MS daughter spectrum (daughters of $m / z 207$ ) are at $m / z$ 118 and 86. Corresponding fragmentation points are shown in Figure 4(C). These are consistent with the $N^{\varepsilon}$-acetylthialysine, but not with the $N^{\alpha}$-acetyl isomer. Minor fragments at $\mathrm{m} / \mathrm{z} 130$ and $\mathrm{m} / \mathrm{z} 44$ suggest a small amount of the latter isomer, but the majority appears to be acetylated on the side chain.
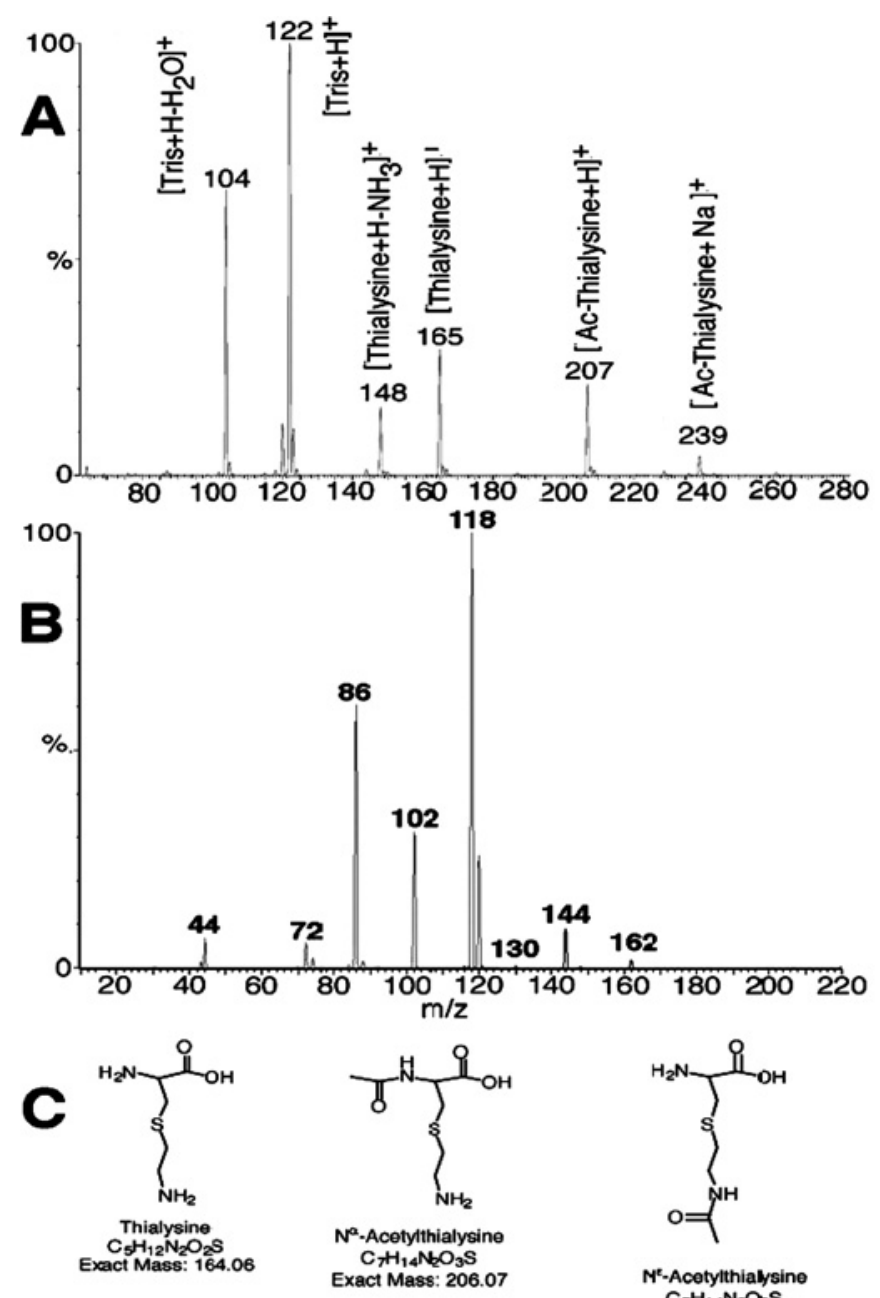

$\mathrm{C}_{7} \mathrm{H}_{14} \mathrm{~N}_{2} \mathrm{O}_{3} \mathrm{~S}$ Ecact Mass: 206.07
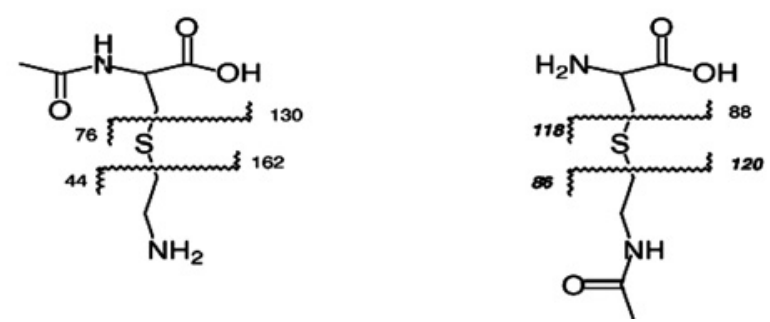

Figure 4 MS analysis of the reaction products formed using thialysine as a substrate for human SSAT2

Reactions containing $1 \mu \mathrm{g}$ of purified hsSSAT2 and $25 \mathrm{mM}$ Tris/HCl, pH 8.0, $10 \mathrm{mM}$ thialysine and $1 \mathrm{mM}$ acetyl-CoA in a total volume of $50 \mu \mathrm{l}$ were incubated at $30^{\circ} \mathrm{C}$ for $1 \mathrm{~h}$ and analysed using MS to determine the nature of the $\mathrm{N}$-acetylthialysine product formed as described in the Materials and methods section. Parallel control reactions included reactions containing inactivated protein and all other components, as well as reactions that contained active enzyme with acetyl-CoA but without thialysine. (A) shows a full-scan electrospray spectrum of product formed with the human SSAT2 enzyme. (B) shows the MS/MS spectrum of daughter ions generated from collision-induced dissociation of $\mathrm{m} / \mathrm{z} 207\left([\mathrm{M}+\mathrm{H}]^{+}\right.$of acetylthialysine). (C) shows the potential fragmentation products from $N^{\alpha}$-acetylthialysine (left) and $N^{\varepsilon}$-acetylthialysine (right).

Further confirmation that the product of the reaction was $N^{\varepsilon}$-acetylthialysine was obtained by separation of the products of the enzyme reaction using reversed-phase HPLC in collaboration with Dr Kai Lüersen at the Bernhard Nocht Institute for Tropical Medicine in Hamburg, Germany. Authentic standards of 

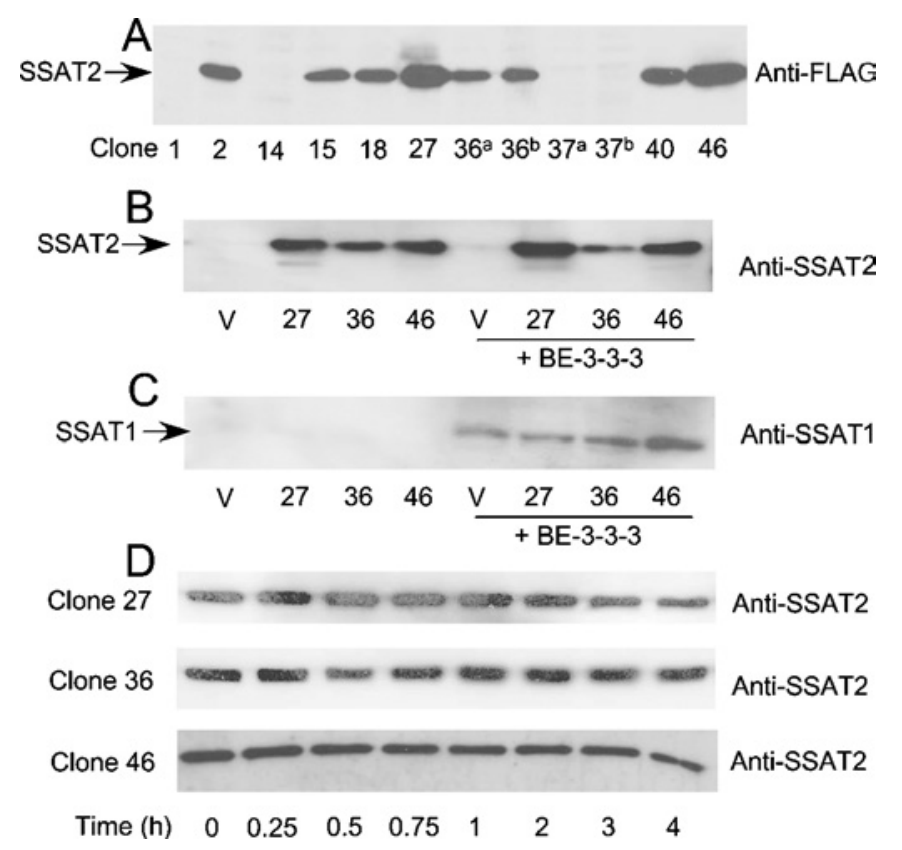

Figure 5 Western blots of cell extracts from NIH-3T3 cells stably expressing human SSAT2 and probed with anti-FLAG, anti-SSAT2 or anti-SSAT1 antibodies

Cells were grown as indicated in the legend to Table 2. Cell extracts were prepared by three freeze-thaw cycles followed by centrifugation at $16000 \boldsymbol{g}_{\mathrm{av}}$ for $20 \mathrm{~min}$ to remove insoluble cellular components. Extracts were resolved by SDS/PAGE ( $15 \%$ gels) and transferred on to a PVDF membrane for immunoblotting as described in the Materials and methods section. (A) shows extracts from a selection of G418-resistant clones numbered in order of isolation and probed with an anti-FLAG antibody. The total amount of protein loaded into each lane of a large gel was $25 \mu \mathrm{g}$ (lanes with a superscript a) or $50 \mu \mathrm{g}$ (lanes with a superscript b) as indicated. (B) shows the SSAT2 protein detected using an anti-SSAT2 antibody in $10 \mu \mathrm{g}$ of total protein from clones 27, 36 and 46 either untreated or treated with $10 \mu \mathrm{M} \mathrm{BE}-3-3-3$ for $48 \mathrm{~h}$, as indicated. Control extracts (V) were from cells transfected with an empty vector. (C) shows the same extracts resolved in (B) that were probed with an anti-SSAT1 antibody. (D) illustrates the levels of SSAT2 protein (probed with an anti-SSAT2 antibody) in clones 27, 36 and 46 after treatment with $0.2 \mathrm{mM}$ cycloheximide for up to $4 \mathrm{~h}$.

$N^{\alpha}$-acetylthialysine and $N^{\varepsilon}$-acetylthialysine were derivatized with OPA prior to injection, and the separated products were well resolved. The OPA-derivatized products from reactions containing either human SSAT2 or the $S$. pombe enzyme incubated in standard assays containing thialysine and $\left[{ }^{14} \mathrm{C}\right]$ acetyl-CoA were detected using a radiochemical detector connected downstream of the fluorescence detector. The resulting retention time of the labelled product from both the human SSAT2 and $S$. pombe enzymes were consistent with acetylation of the $\varepsilon$-amino group of thialysine (results not shown).

\section{Overexpression of SSAT2 in NIH-3T3 cells}

In order to study the effects of increased expression of SSAT2 on cell growth and polyamine metabolism, NIH-3T3 cells were stably transfected with a mammalian expression plasmid that encoded the human SSAT2 enzyme with an N-terminal FLAG epitope tag under the control of the strong CMV IE promoter. A number of G418-resistant clones were isolated, including empty vector controls. Cell extracts were prepared from ten clones, and SSAT2 expression was initially confirmed in seven of these cell lines using an anti-FLAG M5 antibody (Figure 5A). In contrast with the difficulty observed in establishing stable cell lines that overexpressed human SSAT1 [24], the human SSAT2 protein was
Table 2 Polyamine content and spermidine acetyltransferase activity of control NIH-3T3 cells and stable clones that overexpress SSAT2

The stable cell lines listed in the table were derived from NIH-3T3 cells by clonal selection as described in the Materials and methods section. Control (G418-resistant cells transfected with empty vector) and the three individual SSAT2 clones indicated were plated at a density of $2 \times$ $10^{5} \mathrm{cell} / \mathrm{s} / 6 \mathrm{~mm}$ dish and incubated for $6 \mathrm{~h}$ to allow the cells to adhere to the plates. Cells were then treated with or without $10 \mu \mathrm{M} \mathrm{BE}-3-3-3$ and harvested $48 \mathrm{~h}$ later for polyamine and enzyme assays, as described in the Materials and methods section. Polyamine content was determined by HPLC as described previously and values shown are the means \pm S.D. for five replicates. Spermidine acetyltransferase activity was determined using the ${ }^{14} \mathrm{C}$ lacetyl-CoA filter binding assay described previously at a fixed spermidine concentration of $10 \mathrm{mM}$, and values shown are the means \pm S.D. for three replicates.

\begin{tabular}{|c|c|c|c|c|c|}
\hline \multirow[b]{2}{*}{ Cells } & \multirow[b]{2}{*}{ Treatment } & \multicolumn{3}{|c|}{ Polyamine content (nmol/mg of protein) } & \multirow{2}{*}{$\begin{array}{l}\text { Acetyltransferase } \\
\text { activity* (pmol/ } \\
\text { min per mg) }\end{array}$} \\
\hline & & Putrescine & Spermidine & Spermine & \\
\hline Control & None & $4.7 \pm 0.5$ & $22.9 \pm 1.9$ & $8.0 \pm 1.4$ & $7.7 \pm 4.1$ \\
\hline Clone 27 & None & $4.0 \pm 0.5$ & $25.6 \pm 2.6$ & $9.2 \pm 1.3$ & $20.3 \pm 0.8$ \\
\hline Clone 36 & None & $6.4 \pm 0.7$ & $34.2 \pm 2.6$ & $15.0 \pm 1.2$ & $8.0 \pm 0.2$ \\
\hline Clone 46 & None & $9.3 \pm 0.9$ & $34.3 \pm 2.7$ & $10.9 \pm 0.4$ & $9.9 \pm 1.2$ \\
\hline Control & BE-3-3-3 & $6.6 \pm 0.9$ & $9.7 \pm 3.3$ & $<0.5$ & $1429 \pm 55$ \\
\hline Clone 27 & BE-3-3-3 & $7.8 \pm 2.1$ & $12.9 \pm 2.3$ & $1.5 \pm 0.4$ & $2129 \pm 233$ \\
\hline Clone 36 & BE-3-3-3 & $10.9 \pm 1.4$ & $17.9 \pm 2.0$ & $3.5 \pm 0.6$ & $1290 \pm 166$ \\
\hline Clone 46 & BE-3-3-3 & $9.7 \pm 1.1$ & $9.0 \pm 1.1$ & $<0.5$ & $2075 \pm 242$ \\
\hline
\end{tabular}

* Measured with $10 \mathrm{mM}$ spermidine as substrate.

readily expressed to high levels in NIH-3T3 cells. Three cell lines that were selected for further study included clones 27, 36 and 46, to reflect different levels of SSAT2 expression.

A polyclonal antibody was raised in rabbits using the purified human SSAT2 enzyme as immunogen. This antibody was used to examine expression of SSAT2 in the three selected clones, as well as a control cell line that harboured the empty vector. As expected from the results with the anti-FLAG M5 antibody, ectopically expressed SSAT2 was detected in all three cell lines using the anti-SSAT2 antibody, but no signal was detected in extracts from empty vector controls (Figure 5B). This suggests that endogenous expression of SSAT2 is below detection under these conditions, but it cannot be discounted that the antibody may not react with the mouse SSAT2 protein, even though it is $89 \%$ identical with its human counterpart (alignment not shown). SSAT2 expression was unaffected by treatment with the polyamine analogue BE-3-3-3 (Figure 5B), in contrast with endogenous SSAT1, whose level of expression is greatly increased in the same NIH-3T3 cell lines under the same conditions (Figure 5C). SSAT1 was identified with an antiserum raised against human SSAT1 [25]. It appears, from Figures 5(B) and 5(C), that there is no cross-reactivity between the rabbit antibodies to human SSAT1 and SSAT2, with the caveat that the human SSAT2 antibody may or may not react with the mouse protein.

Overexpression of human SSAT2 in NIH-3T3 cells was not detrimental to cell growth. Clones 27, 36 and 46 divided with generation times of $20.8,19.5$ and $18.4 \mathrm{~h}$ respectively compared with $19.3 \mathrm{~h}$ for empty vector control cells. The same cell lines were grown for $48 \mathrm{~h}$ in the absence or presence of $10 \mu \mathrm{M}$ BE-3-3-3, and extracts were assayed for acetyltransferase activity with spermidine as a substrate, and for polyamine content (Table 2). There was no difference in spermidine acetyltransferase activity in untreated control and clone 27, 36 and 46 cells, and this activity was very low in all cases (Table 2). Treatment with BE-3-3-3 caused an increase in spermidine acetyltransferase activity due to induction of endogenous SSAT1 (Figure 5C) that ranged from 
185-fold for control cells to 105-, 161- and 210-fold for clones 27,36 and 46 respectively.

As expected from the known ability of SSAT1 to act as a polyamine catabolic enzyme whose function is associated with decreasing spermidine and spermine levels, all of the cells responded to treatment with BE-3-3-3, with a decrease in spermine and spermidine pools. In contrast, overexpression of human SSAT2 increased, rather than decreased, the total polyamine content of clones 36 and 46, with little change in the polyamine profile of clone 27 when compared with empty vector controls (Table 2). This is not consistent with SSAT2 having a role in metabolizing polyamines, but is in keeping with the high $K_{\mathrm{m}}$ values and low $k_{\text {cat }}$ values determined for polyamines using the purified enzyme (Table 1) and the absence of a detectable increase in the ability of extracts from clones 27,36 and 46 to acetylate spermidine in vitro.

\section{Turnover of human SSAT2 protein}

Previous work showed that human SSAT1 is degraded by the ubiquitin/proteasome pathway in reticulocyte lysates with a halflife of approx. $30 \mathrm{~min}$ under standard assay conditions $[9,10]$. A similar half-life was measured in mammalian cells [24]. To test whether the differences in amino acid sequence between the human SSAT1 and SSAT2 enzymes had any effect on the turnover of the protein, their stability was examined both in reticulocyte lysates and in the stable cell lines 27, 36 and 46.

In order to examine the stability of human SSAT2 in a cellular context, the NIH-3T3 clones that overexpress human SSAT2 were grown for $48 \mathrm{~h}$, before treatment with cycloheximide to inhibit further protein synthesis. Cells were harvested at times up to $4 \mathrm{~h}$ afterwards, and extracts were resolved by SDS/PAGE for analysis of remaining SSAT2 protein by Western blotting. The results shown in Figure 5(D) indicate that SSAT2 was relatively stable over the $4 \mathrm{~h}$ time period examined, in contrast with the published antigenic half-life of $30 \mathrm{~min}$ after cycloheximide treatment observed for SSAT1 in stably transfected CHO cells [24].

When studied in reticulocyte lysates described previously $[9,10],{ }^{35}$ S-labelled SSAT2 was found to be considerably more stable than SSAT1, and showed little accumulation of high-molecular-mass conjugates with ubiquitin in the presence of ubiquitin aldehyde and the proteasome inhibitor MG132 (results not shown).

\section{DISCUSSION}

Comparison of the GNAT family indicates that human SSAT2 is more closely related to SSAT1 than to any other member, but our results show clearly that, despite this similarity, SSAT2 is not likely to be involved in polyamine metabolism. Although acetylation of polyamines can be demonstrated in vitro with large amounts of the recombinant purified SSAT2 protein, the very low activity towards these substrates argues against any physiological role in regulating polyamine content. The results of expression of SSAT2 in NIH-3T3 cells are consistent with this, since there was no decrease in polyamine content and no acetylpolyamines detected. In contrast, expression of comparable levels of SSAT1 (assuming that the immunochemical detection is equally sensitive in both cases) in these cells by induction of the endogenous activity by BE-3-3-3 dramatically reduced the polyamine content.

An alternative explanation for the lack of effect of SSAT2 on polyamine content was suggested by Chen et al. [16], who hypothesized that compartmentation of the expressed SSAT2 might account for a lack of acetylation of cellular polyamines.
Although we cannot absolutely rule out such compartmentation, a far more likely explanation is the very low activity of SSAT2 towards spermidine and spermine as substrates. Our experiments differ from theirs in that we assayed the kinetics of SSAT2 in detail using purified recombinant protein, whereas they only detected activity by measuring acetylation in lysates from HEK-293 cells transiently transfected with plasmids for SSAT1 and SSAT2 using an assay with the potential substrate at a single concentration of $3 \mathrm{mM}$. The inadequacy of this assay is demonstrated by the fact that they found that putrescine, which is not a substrate for purified SSAT1, had a relative activity of $9 \%$ that of spermidine. Extracts from SSAT2-transfected cells in the experiments of Chen et al. [16] had $25 \%$ of the activity with spermidine as a substrate that was seen with extracts from SSAT1-transfected cells, and $55 \%$ of the activity with spermine as a substrate, but these results were not corrected for the amount of protein expressed. Our results indicate that, on the basis of $k_{\text {cat }} / K_{\mathrm{m}}$ values, the SSAT2 activity is actually much less than $0.01 \%$ of the activity of SSAT 1 .

Previous studies using site-directed mutagenesis identified several residues in SSAT1 that are implicated in polyamine substrate binding to the enzyme active site. A notable difference between the aligned sequences is the absence in the human and $S$. pombe SSAT2 proteins of the C-terminal MATEE motif present in SSAT1, which was shown to be important for polyamine acetyltransferase activity and in mediating the stabilizing effects of polyamine analogues $[9,11]$. Another key region of importance with regard to polyamine binding in SSAT1 occurs between Glu $^{152}$-Leu ${ }^{156}$. While Glu ${ }^{152}$ is conserved between human SSAT1 and human SSAT2, specific changes occur at residues 155 and 156 , and neither of these residues are conserved in the $S$. pombe SSAT2. Mutation of $\operatorname{Arg}^{155}$ to alanine in SSAT1 was shown previously to decrease SSAT activity and increase the apparent $K_{\mathrm{m}}$ for spermidine 10-fold [12], whereas a poorly active mutant form of SSAT1, L156F-SSAT, was isolated from Chinese hamster ovary cells selected for resistance to the cellular effects of the polyamine analogue BE-3-3-3 [14]. Since the corresponding residue to Leu ${ }^{156}$ in SSAT1 is Phe ${ }^{156}$ in human SSAT2, this may in part account for the poor affinity of polyamines for the SSAT2 protein.

SSAT1 has been shown to undergo rapid degradation by the ubiquitin/26 S proteasome pathway, and results from mutational analysis have also implicated the C-terminal MATEE sequence in conferring this lability $[9,10]$. The human SSAT2 protein appears to be more stable than human SSAT1, and also lacks the corresponding lysine residue at position 87 that may be a preferred site in SSAT1 for ubiquitination [10]. Taken together, these differences may impart some insight into residues that confer specific properties to the respective proteins. It is interesting that the E. coli spermidine acetyltransferase [26] and the spermidine/spermine acetyltransferase BltD from Bacillus subtilis [27] bear little resemblance to the primary amino acid sequence of human SSAT1. This may indicate the evolution of two distinct structural classes of polyamine acetyltransferases, such as occurs with bacterial and eukaryotic ornithine decarboxylases [28].

Splice variants of SSAT2 may exist as suggested by (a) Figure 2, (b) by the presence of additional sequences in the human EST clone (ID 503372) and (c) a cDNA clone isolated from a human testis library that lacked sequence corresponding to exon 4 (results not shown). Our results show clearly that polyamines are not the normal substrate for SSAT2, but the widespread expression of the mRNA for this enzyme and the presence of a similar enzyme in $S$. pombe and $C$. elegans suggest that it must play some physiological role. Since SSAT2 has a preferential activity towards thialysine, it would be more appropriately described as thialysine $N^{\varepsilon}$-acetyltransferase. The accompanying paper by 
Abo-Dalo et al. [29] reaches the same conclusion based on studies of the equivalent enzyme from $C$. elegans.

A bacterial thialysine $N^{\varepsilon}$-acetyltransferase, with a weak activity towards lysine, was previously identified from Aerobacter aerogenes [30]. This enzyme is inducible by thialysine, and allows growth with this lysine antagonist as the sole nitrogen source. Other enzymes have been isolated from yeast that acetylate lysine at the $N^{\varepsilon}$-position and have activity towards thialysine. These enzymes are preferentially induced by lysine, and are involved in lysine catabolism $[19,31]$.

Metabolites formed from thialysine have been characterized and identified in mammalian tissues including brain [32,33]. The formation of thialysine can occur via the reaction of serine and cysteamine or pantetheine catalysed by cystathionine- $\beta$-synthase [34]. Further metabolism of thialysine by L-amino-acid oxidase $[33,35,36]$ or a mammalian glutamine transaminase $[33,36]$ leads to the cyclic ketimine, AECK [S-(aminoethyl)-Lcysteine ketimine] [32]. AECK can be reduced to form TMA (1,4thiomorpholine-3,5-dicarboxylic acid) by a reductase isolated from bovine brain [37], or it can dimerize spontaneously to form a tricyclic product that loses a carboxy group to form AECKdecarboxylated dimer (AECK-DD) [38,39]. AECK, TMA and AECK-DD have been found in bovine brain, and AECK-DD has been found in human urine and plasma and in a variety of vegetables and human cells [39]. The functions of AECK, TMA and AECK-DD are not known, although the former two compounds have been postulated to serve neurochemical roles, whereas the latter has strong antioxidant properties and could play a role in prevention of damage from hydroxyl radicals, peroxynitrite and other oxidative products. The $N^{\varepsilon}$-acetylation of thialysine would prevent its conversion into AECK, and hence TMA and AECK-DD: the thialysine $N^{\varepsilon}$-acetyltransferase may therefore control this pathway.

This work was supported by grants GM26290 and CA18138 from the National Institutes of Health, Bethesda, MD, U.S.A. We are grateful to Dr Kai Lüersen for helpful discussions and exchanging information, as well as for conducting the HPLC analysis to distinguish the $\mathrm{N}$-acetylthialysine isomer formed in enzyme reactions using the human and $\mathrm{S}$. pombe enzymes. C.S.C. wishes to thank Dr Vincent Chau for the discovery of the human and mouse SSAT2 nucleotide sequences, the construction of bacterial expression systems for SSAT2, the purification of bacterially expressed proteins as described herein, and for supplying purified SSAT2 protein for the present study.

\section{REFERENCES}

1 Pegg, A. E. (1986) Recent advances in the biochemistry of polyamines in eukaryotes. Biochem. J. 234, 249-262

2 Cohen, S. S. (1998) A Guide to the Polyamines, Oxford University Press, New York, NY

3 Wallace, H. M., Fraser, A. V. and Hughes, A. (2003) A perspective of polyamine metabolism. Biochem. J. 376, 1-14

4 Casero, Jr, R. A. and Pegg, A. E. (1993) Spermidine/spermine $N^{1}$-acetyltransferase: the turning point in polyamine metabolism. FASEB J. 7, 653-661

5 Seiler, N. (1987) Functions of polyamine acetylation. Can. J. Physiol. Pharmacol. 65, 2024-2035

6 Coleman, C. S., Pegg, A. E., Megosh, L. C., Guo, Y., Sawicki, J. A. and O'Brien, T. G. (2002) Targeted expression of spermidine/spermine $N^{1}$-acetyltransferase increases susceptibility to chemically induced skin carcinogenesis. Carcinogenesis $\mathbf{2 3}$, $359-364$

7 Casero, Jr, R. A. and Woster, P. M. (2001) Terminally alkylated polyamine analogues as chemotherapeutic agents. J. Med. Chem. 44, 1-26

8 Hahm, H. A., Ettinger, D. S., Bowling, K., Hoker, B., Chen, T. L., Zabelina, Y. and Casero, Jr, R. A. (2002) Phase I study of $N^{1}, N^{11}$-diethylnorspermine in patients with non-small cell lung cancer. Clin. Cancer Res. 8, 684-690

9 Coleman, C. S. and Pegg, A. E. (1997) Proteasomal degradation of spermidine/spermine $N^{1}$-acetyltransferase requires the carboxyl-terminal glutamic acid residues. J. Biol. Chem. 272, 12164-12169
10 Coleman, C. S. and Pegg, A. E. (2001) Polyamine analogues inhibit the ubiquitination of spermidine/spermine $N^{1}$-acetyltransferase and prevent its targeting to the proteasome for degradation. Biochem. J. 358, 137-145

11 Coleman, C. S., Huang, H. and Pegg, A. E. (1995) Role of the carboxyl terminal MATEE sequence of spermidine/spermine $N^{1}$-acetyltransferase in the activity and stabilization by the polyamine analog $N^{1}, N^{12}$-bis(ethyl)spermine. Biochemistry $\mathbf{3 4}$, 13423-13430

12 Coleman, C. S., Huang, H. and Pegg, A. E. (1996) Structure and critical residues at the active site of spermidine/spermine $N^{1}$-acetyltransferase. Biochem. J. 316, 697-701

13 Lu, L., Berkey, K. A. and Casero, Jr, R. A. (1996) RGFGIGS is an amino acid sequence required for acetyl coenzyme $\mathrm{A}$ binding and activity of human spermidine/spermine $N^{1}$-acetyltransferase. J. Biol. Chem. 271, 18920-18924

14 McCloskey, D. E. and Pegg, A. E. (2003) Properties of the spermidine/spermine $N^{1}$-acetyltransferase mutant L156F that decreases cellular sensitivity to the polyamine analogue $N^{1}, N^{11}$-bis(ethyl)norspermine. J. Biol. Chem. 278, 13881-12887

15 Coleman, C. S., Chau, V. and Pegg, A. E. (2001) Identification of a novel polyamine acetylase. FASEB Proceedings A169

16 Chen, Y., Vujcic, S., Liang, P., Diegelman, P., Kramer, D. L. and Porter, C. W. (2003) Genomic identification and biochemical characterization of a second spermidine/spermine $N^{1}$-acetyltransferase. Biochem. J. 373, 661-667

17 Bradford, M. (1976) A rapid and sensitive method for the quantitation of microgram quantities of protein utilizing the principle of protein-dye binding. Anal. Biochem. 72 , 248-254

18 Della Ragione, F. and Pegg, A. E. (1983) Studies of the specificity and kinetics of rat liver spermidine/spermine $N^{1}$-acetyltransferase. Biochem. J. 213, 701-706

19 Bode, R., Thurau, A.-M. and Schmidt, H. (1993) Characterization of acetyl-CoA:L-lysine $N^{6}$-acetyltransferase, which catalyzes the first step of carbon catabolism from lysine in Saccharomyces cerevisiae. Arch. Microbiol. 160, 397-400

20 Pegg, A. E., Wechter, R., Poulin, R., Woster, P. M. and Coward, J. K. (1989) Effect of $S$-adenosyl-1,12-diamino-3-thio-9-azadodecane, a multisubstrate inhibitor of spermine synthase, on polyamine metabolism in mammalian cells. Biochemistry $\mathbf{2 8}$, 8446-8453

21 Coleman, C. S., Hu, G. and Pegg, A. E. (2004) Putrescine biosynthesis in mammalian tissues. Biochem. J. 379, 849-855

22 Xiao, L., Celano, P., Mank, A. R., Griffin, C., Jabs, E. W., Hawkins, A. L. and Casero, Jr, R. A. (1992) Structure of the human spermidine/spermine $N^{1}$-acetyltransferase gene. Biochem. Biophys. Res. Commun. 187, 1493-1502

23 Neuwald, A. F. and Landsman, D. (1997) GCN5-related histone N-acetyltransferases belong to a diverse superfamily that includes the yeast SPT10 protein. Trends Biochem. Sci. 22, 154-155

24 McCloskey, D. E., Coleman, C. S. and Pegg, A. E. (1999) Properties and regulation of human spermidine/spermine $N^{1}$-acetyltransferase stably expressed in Chinese hamster ovary cells. J. Biol. Chem. 274, 6175-6182

25 Casero, Jr, R. A., Gabrielson, E. W. and Pegg, A. E. (1994) Immunohistochemical staining of human spermidine/spermine $N^{1}$-acetyltransferase superinduced in response to treatment with antitumor polyamine analogues. Cancer Res. $\mathbf{5 4}$, $3955-3958$

26 Fukuchi, J., Kashiwagi, K., Takio, K. and Igarashi, K. (1994) Properties and structure of spermidine acetyltransferase in Escherichia coli. J. Biol. Chem. 269, 22581-22585

27 Woolridge, D. P., Martinez, J. D., Stringer, D. E. and Gerner, E. W. (1999) Characterization of a novel spermidine/spermine acetyltransferase, BItD, from Bacillus subtilis. Biochem. J. 340, 753-758

28 Grishin, N. V., Phillips, M. A. and Goldsmith, E. J. (1995) Modeling of the spatial structure of eukaryotic ornithine decarboxylase. Protein Sci. 4, 1291-1304

29 Abo-Dalo, B., Ndjonka, D., Pinnen, F., Liebau, E. and Luersen, K. (2004) A novel member of the GCN5-related N-acetyltransferase superfamily from Caenorhabditis elegans preferentially catalyses the N-acetylation of thialysine [S-(2-aminoethyl)-L-cysteine]. Biochem. J. 383, 129-137

30 Tanaka, H. and Soda, K. (1974) Enzymatic N-acetylation of lysine analogs. J. Biol. Chem. 249, 5285-5289

31 Schmidt, H., Bode, R. and Birnbaum, D. (1988) Lysine degradation in Candida maltosa: occurrence of a novel enzyme, acetyl-COA:L-lysine N-acetyltransferase. Arch. Microbiol. 150, 215-218

32 Cavallini, D., Ricci, G., Dupre, S., Pecci, L., Costa, M., Matarese, R. M., Pensa, B. Antonucci, A., Solinas, S. P. and Fontana, M. (1991) Sulfur-containing cyclic ketimines and imino acids. Eur. J. Biochem. 202, 217-223

33 Cooper, A. J. L. (2004) The role of glutamine transaminase K (GTK) in sulfur and $\alpha$-keto acid metabolism in the brain, and in the possible bioactivation of neurotoxicants. Neurochem. Int. 44, 557-577 
34 Pitari, G., Maurizi, G., Flati, V., Ursini, C. L., Spera, L., Dupre, S. and Cavallini, D. (1992) Enzymatic synthesis of $S$-aminoethyl-L-cysteine from pantetheine.

Biochim. Biophys. Acta 1116, 27-33

35 Cavallini, D., Ricci, G., Federici, G., Costa, M., Pensa, B., Matarese, R. M. and Achilli, M. (1982) The oxidation of sulfur-containing amino acids by L-amino acid oxidases. Adv. Exp. Med. Biol. 148, 359-374

36 Ricci, G., Nardini, M., Federici, G. and Cavallini, D. (1986) The transamination of L-cystathionine, L-cysteine and related compounds by a bovine kidney transaminase. Eur. J. Biochem. 157, 57-63

Received 12 May 2004/8 July 2004; accepted 29 July 2004

Published as BJ Immediate Publication 29 July 2004, DOI 10.1042/BJ20040790
37 Nardini, M., Ricci, G., Vesci, L., Pecci, L. and Cavallini, D. (1988) Bovine brain ketimine reductase. Biochim. Biophys. Acta 957, 286-292

38 Matarese, R. M., Macone, A., Crescentini, G., Dupre, S. and Cavallini, D. (1998) Detection of decarboxylated dimer of aminoethylcysteine ketimine in bovine cerebellum. Neurochem. Int. 32, 365-368

39 Nardini, M., Macone, A. and Matarese, R. M. (2003) Determination of aminoethylcysteine ketimine decarboxylated dimer in human plasma and cultured cells by high-performance liquid chromatography with electrochemical detection. J. Chromatogr. B Biomed. Sci. Appl. 795, 319-327 